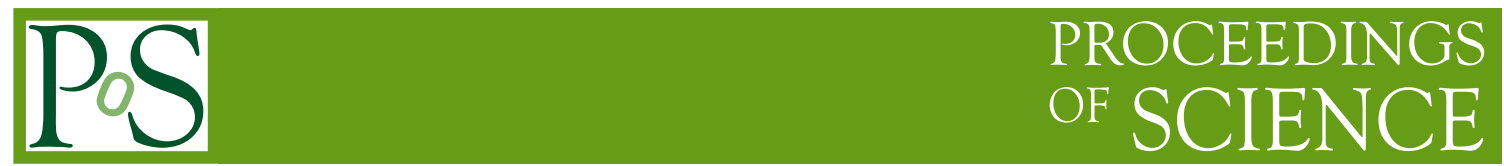

\title{
Particle Number Fluctuations in Statistical Models
}

\section{Viktor Begun*}

Bogolyubov Institute for Theoretical Physics, Kiev, Ukraine.

E-mail: vicl@univ.kiev.ua

The influence of global conservation laws, quantum statistics, resonance decays, and finite detector acceptance on particle number fluctuation is studied. The prediction for the scaled variances of negatively and positively charged hadrons is shown for the most central $\mathrm{Pb}+\mathrm{Pb}(\mathrm{Au}+\mathrm{Au})$ collisions for different collision energies from SIS to LHC.

Correlations and Fluctuations in Relativistic Nuclear Collisions

July 7-9 2006

Florence, Italy

${ }^{*}$ Speaker. 


\section{Introduction}

The statistical approach to strong interactions is surprisingly successful in describing experimental results on hadron production properties in nuclear collisions at high energies (see e.g. Ref. [1], [2], [3] and references therein). This motivates a rapid development of statistical models and it raises new questions, previously not addressed in statistical physics. In particular, an applicability of the models formulated within various statistical ensembles. Recently, it was found that global conservation laws suppress multiplicity fluctuations [4], [5] [6]. This paper illustrates the influence of quantum statistics, resonance decays, and finite detector acceptance on this effect.

\section{Global conservation of charges}

Multiplicity fluctuations can be quantified by the scaled variance. For positively, and negatively, charged particles the scaled variance reads:

$$
\omega^{ \pm} \equiv \frac{\left\langle N_{ \pm}^{2}\right\rangle-\left\langle N_{ \pm}\right\rangle^{2}}{\left\langle N_{ \pm}\right\rangle}
$$

The scaled variance for Poisson distribution is 1 , independently of its mean value:

$$
\omega_{\text {poisson }}^{ \pm}=1
$$

Experimentally, the averaging in the Eq. (2.1) means an averaging on event-by-event basis: a given observable is measured in each collision event and the fluctuations are evaluated for the selected set of these events (see, e.g., review [3]). To calculate a statistical "background" for multiplicity fluctuations one has to choose a statistical ensemble for this calculation: grand canonical (GCE), canonical (CE), or microcanonical (MCE).

As a simplest example, let us consider the system which consists of one sort of positively and negatively charged particles (e.g. $\pi^{+}$and $\pi^{-}$mesons) with total charge equal to zero $Q=0$. In the case of the Boltzmann ideal gas (the interactions and quantum statistics effects are neglected) in the volume $V$ and at temperature $T$ the GCE and CE partition functions read:

$$
\begin{aligned}
Z_{\text {g.c.e. }}(V, T) & =\sum_{N_{+}=0}^{\infty} \sum_{N_{-}=0}^{\infty} \frac{\left(\lambda_{+} z\right)^{N_{+}}}{N_{+} !} \frac{\left(\lambda_{-} z\right)^{N_{-}}}{N_{-} !}=\exp (2 z), \\
Z_{c . e .}(V, T) & =\sum_{N_{+}=0}^{\infty} \sum_{N_{-}=0}^{\infty} \frac{\left(\lambda_{+} z\right)^{N_{+}}}{N_{+} !} \frac{\left(\lambda_{-} z\right)^{N_{-}}}{N_{-} !} \delta\left(N_{+}-N_{-}\right)= \\
& =\frac{1}{2 \pi} \int_{0}^{2 \pi} d \phi \exp \left[z\left(\lambda_{+} e^{i \phi}+\lambda_{-} e^{-i \phi}\right)\right]=I_{0}(2 z),
\end{aligned}
$$

where $z$ is a single particle partition function:

$$
z=\frac{g V}{2 \pi^{2}} \int_{0}^{\infty} p^{2} d p e^{-\frac{\sqrt{p^{2}+m^{2}}}{T}}=\left\langle N_{ \pm}\right\rangle,
$$

$g$ is a degeneracy factor (number of spin states), $m$ - particle mass. The average values in both the GCE and CE can be calculated as follows:

$$
\left\langle N_{ \pm}\right\rangle \equiv\left[\frac{1}{Z} \lambda_{ \pm} \frac{\partial Z}{\partial \lambda_{ \pm}}\right]_{\lambda_{ \pm}=1}, \quad\left\langle N_{ \pm}^{2}\right\rangle \equiv\left[\frac{1}{Z} \lambda_{ \pm} \frac{\partial}{\partial \lambda_{ \pm}}\left(\lambda_{ \pm} \frac{\partial Z}{\partial \lambda_{ \pm}}\right)\right]_{\lambda_{ \pm}=1} .
$$


In thermodynamic limit, $V \rightarrow \infty$, it gives:

$$
\begin{array}{rlrl}
\left\langle N_{ \pm}\right\rangle & =z, & \left\langle N_{ \pm}^{2}\right\rangle & =z+z^{2}, \\
\left\langle N_{ \pm}\right\rangle_{\text {c.e. }} & \cong z\left(1-\frac{1}{4 z}\right), & \left\langle N_{ \pm}^{2}\right\rangle_{\text {c.e. }} \cong z^{2}
\end{array}
$$

It then follows that the scaled variance in this system in the $\mathrm{CE}$ is two times smaller than in the GCE [4]:

$$
\begin{gathered}
\omega_{\text {g.c.e. }}^{ \pm} \equiv \frac{\left\langle N_{ \pm}^{2}\right\rangle-\left\langle N_{ \pm}\right\rangle^{2}}{\left\langle N_{ \pm}\right\rangle}=1, \\
\omega_{\text {c.e. }}^{ \pm} \equiv \frac{\left\langle N_{ \pm}^{2}\right\rangle_{\text {c.e. }}-\left\langle N_{ \pm}\right\rangle_{\text {c.e. }}^{2}}{\left\langle N_{ \pm}\right\rangle_{\text {c.e. }}}=\frac{1}{2} .
\end{gathered}
$$

The same result can be obtained using the saddle point expansion technique [8] for quantum ideal gas where the scaled variance is also two times smaller in the CE than in the GCE [6]:

$$
\omega_{\text {c.e. }}^{ \pm}=\frac{1}{2} \omega_{\text {g.c.e. }}^{ \pm}\left[1+\mathscr{O}\left(V^{-1}\right)\right]
$$

Exact energy conservation additionally suppress the scaled variance. It means that the thermodynamical equivalence for mean particle number does not apply to fluctuations measured in terms of the scaled variance [4], [5]:

$$
\begin{aligned}
& \langle N\rangle \simeq\langle N\rangle_{\text {c.e. }} \simeq\langle N\rangle_{\text {m.c.e. }}, \\
& \omega_{\text {g.c.e. }} \neq \omega_{\text {c.e. }} \neq \omega_{\text {m.c.e. }}, \quad V \rightarrow \infty
\end{aligned}
$$

Let us consider the fluctuations in the ideal relativistic gas with different types of hadrons in the MCE with exactly fixed the global electric (Q), baryon (B), and strange (S) charges of the statistical system. The system of non-interacting Bose or Fermi particles of species $i$ can be characterized by the occupation numbers $n_{p, i}$ of single quantum states labelled by momenta $p$. The occupation numbers run over $n_{p, i}=0,1$ for fermions and $n_{p, i}=0,1,2, \ldots$ for bosons. The GCE average values and fluctuations of $n_{p, i}$ equal the following:

$$
\begin{aligned}
\left\langle n_{p, i}\right\rangle & =\frac{1}{\exp \left[\left(\sqrt{p^{2}+m_{i}^{2}}-\mu_{i}\right) / T\right]-\gamma_{i}}, \\
v_{p, i}^{2} & \equiv\left\langle\Delta n_{p, i}^{2}\right\rangle \equiv\left\langle\left(n_{p, i}-\left\langle n_{p, i}\right\rangle\right)^{2}\right\rangle=\left\langle n_{p, i}\right\rangle\left(1+\gamma_{i}\left\langle n_{p, i}\right\rangle\right) .
\end{aligned}
$$

In Eq. (2.15), $T$ is the system temperature, $m_{i}$ is the mass of $i$-th particle species, $\gamma_{i}$ corresponds to different statistics $\left(+1\right.$ and -1 for Bose and Fermi, respectively, and $\gamma_{i}=0$ gives the Boltzmann approximation), and chemical potential $\mu_{i}$ equals:

$$
\mu_{i}=q_{i} \mu_{Q}+b_{i} \mu_{B}+s_{i} \mu_{S}
$$

where $q_{i}, b_{i}, s_{i}$ are the electric charge, baryon number and strangeness of particle of species $i$, respectively, while $\mu_{Q}, \mu_{B}, \mu_{S}$ are the corresponding chemical potentials which regulate the average values of these global conserved charges in the GCE. 
The average number of particles of species $i$, the number of positively and negatively charged particles are equal:

$$
\left\langle N_{i}\right\rangle \equiv \sum_{p}\left\langle n_{p, i}\right\rangle=\frac{g_{i} V}{2 \pi^{2}} \int_{0}^{\infty} p^{2} d p\left\langle n_{p, i}\right\rangle, \quad\left\langle N_{+}\right\rangle=\sum_{i, q_{i}>0}\left\langle N_{i}\right\rangle, \quad\left\langle N_{-}\right\rangle=\sum_{i, q_{i}<0}\left\langle N_{i}\right\rangle,
$$

where $g_{i}$ is the degeneracy factor of particle of species $i$. A sum of the momentum states means the momentum integral, which holds in the thermodynamic limit $V \rightarrow \infty$.

Particle number fluctuations and correlations can be calculated in all ensembles using the microscopic correlator method.

$$
\left\langle\Delta N_{i} \Delta N_{j}\right\rangle_{\ldots}=\sum_{p, k}\left\langle\Delta n_{p, i} \Delta n_{k, j}\right\rangle_{\ldots},
$$

where \langle\rangle .. means GCE, CE, or MCE microscopic correlator. The scaled variances of negatively and positively charged particles read:

$$
\omega^{-}=\frac{\left\langle\left(\Delta N_{-}\right)^{2}\right\rangle}{\left\langle N_{-}\right\rangle}, \quad \omega^{+}=\frac{\left\langle\left(\Delta N_{+}\right)^{2}\right\rangle}{\left\langle N_{+}\right\rangle},
$$

where

$$
\left\langle\left(\Delta N_{-}\right)^{2}\right\rangle=\sum_{i, j ; q_{i}<0, q_{j}<0}\left\langle\Delta N_{i} \Delta N_{j}\right\rangle, \quad\left\langle\left(\Delta N_{+}\right)^{2}\right\rangle=\sum_{i, j ; q_{i}>0, q_{j}>0}\left\langle\Delta N_{i} \Delta N_{j}\right\rangle .
$$

The microscopic correlator in the GCE reads:

$$
\left\langle\Delta n_{p, i} \Delta n_{k, j}\right\rangle=v_{p, i}^{2} \delta_{i j} \delta_{p k}
$$

where $v_{p, i}^{2}$ is given by Eq. (2.16). This gives a possibility to calculate the fluctuations of different observables in the GCE. Note that only particles of the same species, $i=j$, and on the same level, $p=k$, do correlate in the GCE. Thus, Eq. (2.22) is equivalent to Eq. (2.16): only the Bose and Fermi effects for the fluctuations of identical particles on the same level are relevant in the GCE.

The MCE microscopic correlator is as follows [6], [7]:

$$
\begin{aligned}
& \left\langle\Delta n_{p, i} \Delta n_{k, j}\right\rangle_{m . c . e .}=v_{p, i}^{2} \delta_{i j} \delta_{p k}-\frac{v_{p, i}^{2} v_{k, j}^{2}}{|A|}\left[q_{i} q_{j} M_{q q}+b_{i} b_{j} M_{b b}+s_{i} s_{j} M_{s s}\right. \\
& +\left(q_{i} s_{j}+q_{j} s_{i}\right) M_{q s}-\left(q_{i} b_{j}+q_{j} b_{i}\right) M_{q b}-\left(b_{i} s_{j}+b_{j} s_{i}\right) M_{b s} \\
& \left.+\varepsilon_{p i} \varepsilon_{k j} M_{\varepsilon \varepsilon}-\left(q_{i} \varepsilon_{p j}+q_{j} \varepsilon_{k i}\right) M_{q \varepsilon}+\left(b_{i} \varepsilon_{p j}+b_{j} \varepsilon_{k i}\right) M_{b \varepsilon}-\left(s_{i} \varepsilon_{p j}+s_{j} \varepsilon_{k i}\right) M_{s \varepsilon}\right],
\end{aligned}
$$

where $|A|$ is the determinant and $M_{i j}$ are the minors of the following matrix:

$$
A=\left(\begin{array}{llll}
\Delta\left(q^{2}\right) & \Delta(b q) & \Delta(s q) & \Delta(\varepsilon q) \\
\Delta(q b) & \Delta\left(b^{2}\right) & \Delta(s b) & \Delta(\varepsilon b) \\
\Delta(q s) & \Delta(b s) & \Delta\left(s^{2}\right) & \Delta(\varepsilon s) \\
\Delta(q \varepsilon) & \Delta(b \varepsilon) & \Delta(s \varepsilon) & \Delta\left(\varepsilon^{2}\right)
\end{array}\right)
$$

with the elements, $\Delta\left(q^{2}\right) \equiv \sum_{p, k} q_{k}^{2} v_{p, k}^{2}, \Delta(q b) \equiv \sum_{p, k} q_{k} b_{k} v_{p, k}^{2}, \Delta(q \varepsilon) \equiv \sum_{p, k} q_{k} \varepsilon_{p k} v_{p, k}^{2}$, etc. The sum, $\sum_{p, k}$, means integration over momentum $p$, and summation over all hadron-resonance species $k$ contained in the model. Note that the presence of MCE terms containing single particle energies, $\varepsilon_{p i}=\sqrt{p^{2}+m_{j}^{2}}$, in the last line of Eq.(2.23) is a consequence of exact energy conservation. In the CE, only charges are conserved exactly, thus the terms of the last line in Eq. (2.23) are absent, and $A$ in Eq. (2.24) becomes the $3 \times 3$ matrix (see Ref. [6]). 


\section{Effect of resonance decays}

The average number of $i$-particles in the presence of primary particles $N_{i}^{*}$ and different resonance types $R$ is the following:

$$
\left\langle N_{i}\right\rangle=\left\langle N_{i}^{*}\right\rangle+\sum_{R}\left\langle N_{R}\right\rangle \sum_{r} b_{r}^{R} n_{i, r}^{R} \equiv\left\langle N_{i}^{*}\right\rangle+\sum_{R}\left\langle N_{R}\right\rangle\left\langle n_{i}\right\rangle_{R}
$$

The summation $\sum_{R}$ runs over all types of resonances. The $\langle\ldots\rangle$ and $\langle\ldots\rangle_{R}$ correspond to the GCE averaging, and that over resonance decay channels. Resonance decay has a probabilistic character. This itself causes the particle number fluctuations in the final state. In the GCE the final state correlators can be calculated as [9]:

$$
\left\langle\Delta N_{i} \Delta N_{j}\right\rangle=\left\langle\Delta N_{i}^{*} \Delta N_{j}^{*}\right\rangle+\sum_{R}\left[\left\langle\Delta N_{R}^{2}\right\rangle\left\langle n_{i}\right\rangle_{R}\left\langle n_{j}\right\rangle_{R}+\left\langle N_{R}\right\rangle\left\langle\Delta n_{i} \Delta n_{j}\right\rangle_{R}\right]
$$

where $b_{r}^{R}$ is the branching ratio of the $r$-th branch, $n_{i, r}^{R}$ is the number of $i$-th particles produced in that decay mode, and $r$ runs over all branches with the requirement $\sum_{r} b_{r}^{R}=1$ and $\left\langle\Delta n_{i} \Delta n_{j}\right\rangle_{R} \equiv$ $\sum_{r} b_{r}^{R} n_{i, r}^{R} n_{j, r}^{R}-\left\langle n_{i}\right\rangle_{R}\left\langle n_{j}\right\rangle_{R}$. Note that different branches are defined in a way that final states with only stable (with respect to strong and electromagnetic decays) hadrons are counted.

All primary particles and resonances become to correlate in the presence of exact charge conservation laws. Thus for the MCE correlators we obtain a new result [6]:

$$
\begin{aligned}
& \left\langle\Delta N_{i} \Delta N_{j}\right\rangle_{m . c . e .}=\left\langle\Delta N_{i}^{*} \Delta N_{j}^{*}\right\rangle_{m . c . e .}+\sum_{R}\left\langle N_{R}\right\rangle\left\langle\Delta n_{i} \Delta n_{j}\right\rangle_{R}+\sum_{R}\left\langle\Delta N_{i}^{*} \Delta N_{R}\right\rangle_{m . c . e .}\left\langle n_{j}\right\rangle_{R} \\
& +\sum_{R}\left\langle\Delta N_{j}^{*} \Delta N_{R}\right\rangle_{m . c . e .}\left\langle n_{i}\right\rangle_{R}+\sum_{R, R^{\prime}}\left\langle\Delta N_{R} \Delta N_{R^{\prime}}\right\rangle_{m . c . e .}\left\langle n_{i}\right\rangle_{R}\left\langle n_{j}\right\rangle_{R^{\prime}} .
\end{aligned}
$$

Additional terms in Eq. (3.3) compared to Eq. (3.2) are due to the correlations induced by exact charge conservations in the MCE. The Eq. (3.3) remains valid in the CE too with $\langle\ldots\rangle_{\text {m.c.e. }}$ replaced by $\langle\ldots\rangle_{c . e .}$, the difference between them appears only when one specifies the microscopic correlators (2.23) of the MCE or CE.

\section{Scaled variances along the chemical freeze-out line}

Mean hadron multiplicities in heavy ion collisions at high energies can be approximately fitted by the GCE hadron-resonance gas model. The fit parameters are temperature $T$, chemical potentials $\left(\mu_{B}, \mu_{S}, \mu_{Q}\right)$, and strangeness suppression factor $\gamma_{S}$, which allows for non-equilibrium strange hadron yields. There are several programs designed for the analysis of particle multiplicities in relativistic heavy-ion collisions within the hadron-resonance gas model, see e.g., SHARE [10], THERMUS [11] and THERMINATOR [12]. In this paper an extended version of the THERMUS thermal model framework [11] is used.

For the chemical freeze-out condition we choose the average energy per particle $\langle E\rangle /\langle N\rangle=$ $1 \mathrm{GeV}$ [13]. Using the standard parametrization [2] we obtain the $T-\mu_{B}$ freeze-out line for central A+A collisions (see Fig. 1).

The center of mass nucleon-nucleon energies, $\sqrt{S_{N N}}$, marked in the figures below correspond to the beam energies at SIS (2A GeV), AGS (11.6A GeV), SPS (20A, 30A, 40A, 80A, and 158A 


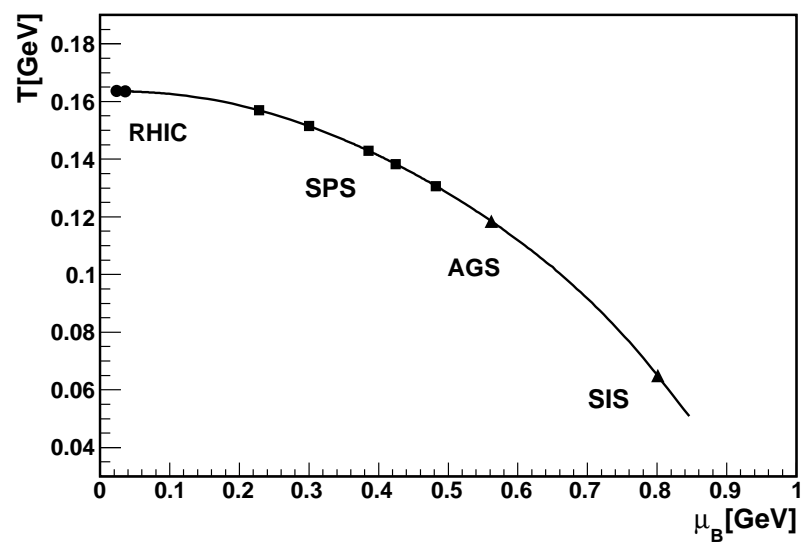

Figure 1: The chemical freeze-out line in central $A+A$ collisions.

$\mathrm{GeV})$, colliding energies at RHIC $\left(\sqrt{S_{N N}}=62.4 \mathrm{GeV}, 130 \mathrm{GeV}\right.$ and $\left.200 \mathrm{GeV}\right)$ and LHC $\left(\sqrt{S_{N N}}=\right.$ $5500 \mathrm{GeV}$ ).

Figure 2 show the prediction for the scaled variances for negatively and positively charged particles as a function of $\sqrt{s_{N N}}$.
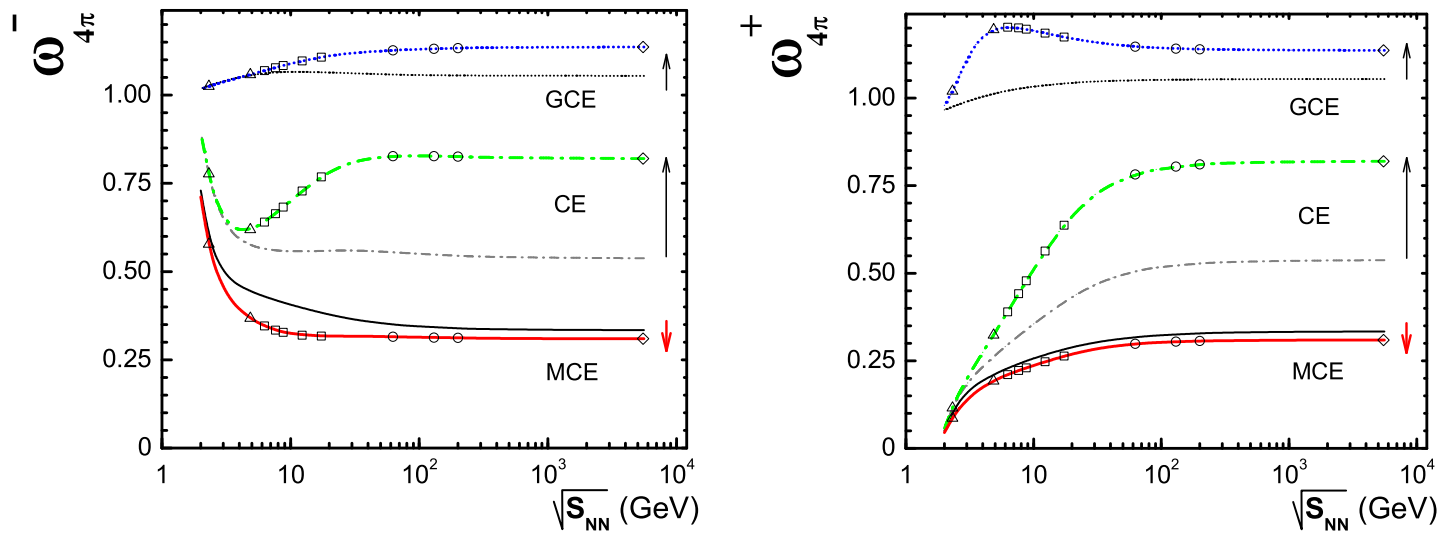

Figure 2: The scaled variances for negatively and positively charged particles, both primordial and final, along the chemical freeze-out line for central $\mathrm{Pb}+\mathrm{Pb}(\mathrm{Au}+\mathrm{Au})$ collisions. Different lines present the GCE, $\mathrm{CE}$, and MCE results. Symbols at the $\mathrm{CE}$ and MCE lines for the final particles correspond to the specific collision energies. The arrows show the effect of resonance decays.

The prediction can be compared with the preliminary NA49 data on $\mathrm{Pb}+\mathrm{Pb}$ collisions at $20 \mathrm{~A}-$ $158 \mathrm{~A} \mathrm{GeV} \mathrm{[14]} \mathrm{using} \mathrm{the} \mathrm{following} \mathrm{approximate} \mathrm{formula:}$

$$
\omega_{\text {acc }}^{ \pm}=1-q+q \omega_{4 \pi}^{ \pm},
$$

where $\omega_{4 \pi}$ refers to an ideal detector with full $4 \pi$-acceptance and $\omega_{a c c}^{ \pm}$is the scaled variance measured by a real detector with a limited acceptance), $\mathrm{q}$ is the ratio between mean multiplicities of 
accepted particles and all hadrons. In the limit of a very 'bad' (or 'small') detector, $q \rightarrow 0$, all scaled variances approach linearly to 1 , i.e., this would lead to the Piossonian distributions for detected particles. However, we find a strong qualitative difference between the predictions of the statistical model valid for any freeze-out conditions and experimental acceptances: the CE and MCE correspond to $\omega_{m . c . e .}^{ \pm}<\omega_{\text {c.e. }}^{ \pm}<1$, and the GCE to $\omega_{\text {g.c.e. }}^{ \pm}>1$.
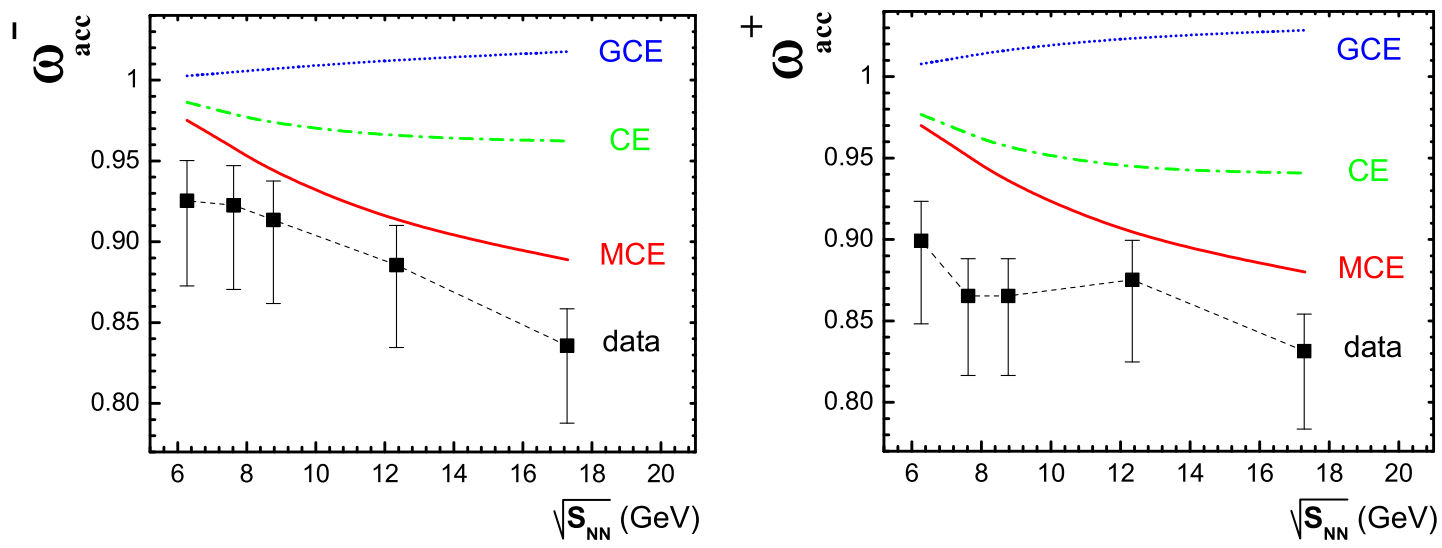

Figure 3: The scaled variances for negative (left) and positive (right) hadrons along the chemical freeze-out line for central $\mathrm{Pb}+\mathrm{Pb}$ collisions at the SPS energies. The corresponding $T$ and $\mu_{B}$ values at different SPS collision energies are presented in Fig. 1. Different lines show the GCE, CE, and MCE results calculated with the NA49 experimental acceptance.

From Fig. 3 it follows that the NA49 data for $\omega^{ \pm}$extracted from the most central $\mathrm{Pb}+\mathrm{Pb}$ collisions at all SPS energies are close to the results of the hadron-resonance gas statistical model within the MCE. The data reveal even stronger suppression of the particle number fluctuations. A possible reason of this is an uncertainty in the determination of the detector acceptance and an additional suppression due to momentum conservation and the excluded volume effects in the hadron-resonance gas.

\section{Summary}

Scaled variances are different in different statistical ensembles, even in thermodynamic limit. The analytical formula for the resonance decay contribution in CE and MCE has been shown. The prediction for the energy dependence of the scaled variances in the most central $\mathrm{Pb}+\mathrm{Pb}$ collisions has been done. A comparison of the statistical model for hadron-resonance gas in CE and MCE with NA49 data was made. The MCE results are the closest to the measured scaled variances for positively and negatively charged particles.

\section{References}

[1] P. Braun-Munzinger, K. Redlich, and J. Stachel, Review for Quark Gluon Plasma 3, eds. R.C. Hwa and X.-N. Wang, World Scientific, Singapore, 2004, [nucl-th/0304013].

[2] J. Cleymans, H. Oeschler, K. Redlich, and S. Wheaton, Phys. Rev. C 73, 034905 (2006); F. Becattini, J. Manninen, and M. Gazdzicki, Phys. Rev. C 73, 044905 (2006). 
[3] H. Heiselberg, Phys. Rep. 351, 161 (2001); S. Jeon and V. Koch, Review for Quark-Gluon Plasma 3, eds. R.C. Hwa and X.-N. Wang, World Scientific, Singapore, 430-490 (2004), [hep-ph/ 0304012 ].

[4] V.V. Begun, M. Gazdzicki, M.I. Gorenstein, and O.S. Zozulya, Phys. Rev. C 70, 034901 (2004).

[5] V.V. Begun, M.I. Gorenstein, and O.S. Zozulya, Phys. Rev. C 72, 014902 (2005); A. Keranen, F. Becattini, V.V. Begun, M.I. Gorenstein, and O.S. Zozulya, J. Phys. G 31, S1095 (2005); F. Becattini, A. Keranen, L. Ferroni, and T. Gabbriellini, Phys. Rev. C 72, 064904 (2005); V.V. Begun, M.I. Gorenstein, A.P. Kostyuk, and O.S. Zozulya, Phys. Rev. C 71, 054904 (2005); J. Cleymans, K. Redlich, and L. Turko, Phys. Rev. C 71, 047902 (2005); J. Phys. G 31, 1421 (2005); V.V. Begun, M.I. Gorenstein, A.P. Kostyuk, and O.S. Zozulya, J. Phys. G 32, 935 (2006). V.V. Begun and M.I. Gorenstein, Phys. Rev. C 73, 054904 (2006).

[6] V.V. Begun, M.I. Gorenstein, M. Hauer, V.P. Konchakovski, and O.S. Zozulya, Phys. Rev. C 74, 044903 (2006).

[7] V.V. Begun, M. Gaździcki, M.I. Gorenstein, M. Hauer, V.P. Konchakovski, B. Lungwitz, in preparation.

[8] F.Becattini, Z.Phys., C 69, (1996); F.Becattini, U.Heinz, ibid. 76, (1997); F.Becattini, A.Keranen, L.Ferroni, T.Gabrielini, Phys.Rev., C 72, (2006).

[9] S. Jeon and V. Koch, Phys. Rev. Lett. 83, 5435 (1999).

[10] G. Torrieri, S. Steinke, W. Broniowski, W. Florkowski, J. Letessier and J. Rafelski, Comput. Phys. Commun. 167 (2005) 229

[11] S. Wheaton, J. Cleymans, J. Phys. G 31 1069-1074 (2005)

[12] A. Kisiel, T. Taluc, W. Broniowski and W. Florkowski, Comput. Phys. Commun. 174, 669 (2006)

[13] J. Cleymans and K. Redlich, Phys. Rev. Lett. 81, 5284 (1998).

[14] B. Lungwitz et al., [for the NA49 Collaboration], in proceedings of Correlations and Fluctuations in Relativistic Nuclear Collisions, July 7-9, 2006, Florence, Italy. 